\title{
Chlamydia Trachomatis Infections: A Hidden Cause of Meibomian Gland Dysfunction
}

\section{Himanshu $\mathrm{SB}^{1}$, Gita $\mathrm{S}^{2}$, Sharma $\mathrm{A}^{1}$ and Pushker $\mathrm{N}^{3}$}

${ }^{1}$ Department of Ocular Microbiology, Dr. R. P. Centre for Ophthalmic Sciences, All India Institute of Medical Sciences, India

2Department of Ocular Microbiology, Dr. R. P. Centre for Ophthalmic Sciences and prof. \& Head deptt. of Microbiology, All India Institute of Medical Sciences, India

${ }^{3}$ Department of Opthalmology, Dr. R. P. Centre for Ophthalmic Sciences, All India Institute of Medical Sciences, India

*Corresponding author: Gita Satpathy, Department of Ocular Microbiology, Dr. R. P. Centre for Ophthalmic Sciences and prof. \& Head dept. of Microbiology, All India Institute of Medical Sciences, New Delhi-110029, India, Tel: 01126593171; Email: gita.satpathy@gmail.com

\section{Abstract}

Purpose: Meibomian gland dysfunction (MGD) is a common eye problem, which is asymptomatic in the early stage, but if left untreated for a prolonged period can exacerbate to dry eye syndrome. Role of bacteria in the pathophysiology of MGD is always controversial. This pilot study was undertaken to find the association of Chlamydia trachomatis apart from the normal biota of the eyelid with MGD in a tertiary care hospital in India.

Methods: A total of 112 MGD patients with follicular conjunctival inflammation with either of the symptoms such as; Meibomitis (22), Dry eye (42), Watering (19), Blepharitis (9), Chalazion/ Hardoleum (20) were referred to the Chlamydial laboratory for further investigation. Conjunctival swabs of both eyes were subjected to direct immunofluorescence assay (DFA) for C. trachomatis antigen detection.

Results: Of the 112 MGD patients, 62 (55.30\%) were found positive for C. trachomatis with DFA, out of which 54(87\%) had good clinical response for topical azithromycin (1\% eye drop), topical lubricants and warm compresses.

Conclusions: We concluded from this study that, C. trachomatis is one of the causes for MGD with follicular conjunctival inflammation and topical azithromycin is a good choice for treatment and improving the signs and symptoms of the infection. As the role of chlamydia in MGD is not clear, this study will disclose an exciting fact about the pathophysiology of the disease, which will in turn improve treatment strategy to some extent to combat with the common but frustrating eye problem.

Keywords: Chlamydia Trachomatis; Meibomian Gland Dysfunction; Blepharitis; Dry Eye; Azithromycin; Direct Fluorescence Antigen DFA 
Abbreviations: MGD: Meibomian Gland Dysfunction; OSDI: Ocusclar Surface Disease Index; MMPs: Matrix Metallo Proteinases; DFA: Direct Immune Fluorescence Assay; FITC: Fluoresceine-Isothiocynate.

\section{Introduction}

Meibomian gland dysfunction (MGD) is a chronic diffuse abnormality of the meibomian glands, which is asymptomatic in the early stage, but if left untreated for a prolonged period can exacerbate to the dry eye syndromes, clinically apparent inflammations and ocular surface diseases. In the early stage, MGD is often overlooked by the Opthalmologists due to its similar signs and symptoms with other eye infections such as; inflammations, irritation, qualitative/ quantitative changes in the secretion of oil from meibomian glands etc $[1,2]$. As per some earlier reports, up to $70 \%$ of people across the world suffer from MGD and it is the leading cause of dry eye disease [3]. As per ocusclar surface disease index (OSDI) data, the prevalence of dry eye ranges from $5 \%$ to $35 \%$ worldwide and $29.25 \%$ in India [4]. Till today, clinicians and researchers don't have a complete understanding on the underlying mechanism, epidemiology, pathophysiology, and management of MGD, therefore several studies are being carried out across the world to find the hidden cause and mechanism behind its occurrence.

MGD is a chronic abnormality of the meibomian glands that occurs either due to blockage or infections of the glands, so that they stop secreting enough oil over the ocular surface. As oil is the major component of the tear film, MGD at the later stage leads to the evaporative dry eye syndrome or severe eyelid problem like blepharitis [5]. As the role of several bacteria in the pathophysiology of MGD is always controversial, there are some studies which correlate MGD with some bacterial colonization [6]. Some studies demonstrated the role of Staphylococcus epidermidis, Staphylococcus aureus, Propioni bacterium with blepharitis, which indicates the possibility that microbes find an altered eyelid environment in MGD more suitable than the normal eyelid $[7,8]$. These bacteria may have a direct or indirect effects on the meibomian gland function such as production of toxic bacterial products(direct effect) or effects on homeostasis of the ocular surface, such as secretion of matrix metallo proteinases (MMPs), cytokine balance and macrophage function(indirect effect) [9]. As per the theory is concerned, for an antibiotic to be completely beneficial in the treatment of a disease, it must be effective against the pathogen most likely to be present in the infected area. Hence detection of the exact pathogen responsible for MGD is very essential for the treatment and management of MGD.

This pilot study was undertaken to know the association of Chlamydia trachomatis apart from the normal biota of the eyelid with MGD in a tertiary care hospital in India.

\section{Material and Methods}

\section{Patient Selection and Collection of Clinical Specimens}

In a retrospective study, conjunctival swabs were collected by the Ophthalmologists(after taking consent from the patients following the ethical guidelines of the Institute) from superior/ inferior palpebral conjunctiva of both the eyes of 112 patients ( 57 females, 55 males; age group 5-65 Yrs; median age: 32.5 Yrs) clinically suspected of MGD with follicular conjunctival inflammation with signs and symptoms of dryness, meibomitis, blepharitis, watering, redness, foreign body sensation, chalazion/ Hardoleum reporting to the outpatient department of Dr RP. Centre for Ophthalmic Sciences, New Delhi, India. Patients with ocular surgery, contact lens uses, eye disorders affecting the ocular surface, infectious conjunctivitis, history of pre-topical antibiotic treatments or systemic steroids use were excluded from this study. Prior to referring the patients to the Chlamydia laboratory, experienced ophthalmologists performed clinical examination using slit lamp biomicroscopy of the anterior segment of the eyes of the 112 patients suspected of having MGD, 22 were with the symptoms of meibomitis, 42 with dry eyes, 19 with watering from eyes, 9 with blepharitis and 20 with chalazion/ hardoleum (Table 1).

Conjunctival swabs were collected with sterile wet cotton swabs and rolled on the clean teflon-coated glass slide to prepare the smear (one specimen from each eye). Slides were prepared in duplicates for each sample collected from the patients following the protocol mentioned above. Slides were air-dried, fixed in cold acetone for 10 minutes and subjected to direct immune fluorescence assay (DFA) for chlamydia antigen detection [10]. As the study was focused on to know the association of only Chlamydia with MGD; hence samples were not processed to identify the presence of other bacterial flora (apart from C. trachomatis) with this infection. 


\section{Open Access Journal of Ophthalmology}

\begin{tabular}{|c|c|}
\hline Age Group of Patients (Yrs) & No. Of Samples \\
\hline $0-10$ & 6 \\
\hline $11-20$ & 26 \\
\hline $21-30$ & 43 \\
\hline $31-40$ & 33 \\
\hline $41-50$ & 30 \\
\hline $51-60$ & 26 \\
\hline $61-70$ & 13 \\
\hline $71-80$ & 05 \\
\hline $81-90$ & 01 \\
\hline
\end{tabular}

Table 1: Trachomatis antigen positivity in MGD patients.

\section{Direct Immune Fluorescence Assay}

Direct immune fluorescence assay (DFA) a CDC approved test was performed with the monoclonal antibody based $C$. trachomatis direct specimen kit (MicroTrak, USA) following the standard protocol mentioned in the kit [10]. Briefly, conjunctival smears were covered with $30 \mu \mathrm{l}$ of fluoresceine-isothiocynate (FITC)-conjugated murine monoclonal antibodies to $C$. trachomatis for 30 minutes at $37^{\circ} \mathrm{C}$ in a humidified chamber. The slides were washed with double distilled water, air-dried, mounted and observed under the fluorescent microscope (Nikon, Japan) with 100X objective. Fixed mammalian cells containing chlamydia elementary/reticulate bodies (EB/RB) (provided with the kit) were used as the positive-control while normal uninfected mammalian cells were used as the negative control in this pilot study. C. trachomatis positive cases were treated with topical azithromycin antibiotic.

\section{Statistical Analysis}

Percentage signifies the antigen positivity. P-value was calculated with Chi-square test (Graph pad software) among the male and female MGD patients. Confidence of Interval was calculated with the formula $\mathrm{CI}=$ Sample mean $\pm 1.96 \times \mathrm{SD} /$ root of sample size (95\% confidence level). Degree of freedom was calculated as sample size -1 .

\section{Results}

Of the 112 patients ( 57 females, 55 males; age $4-65$ Yr) of MGD with conjuctival follicles, 62 (55.35\%) were found positive for C. trachomatis antigen with DFA. Mean age of the patients was 33.77 years. Of the 22 patients of having symptoms of meibomitis, 16(72.7\%) were found positive, of the 42 patients with symptoms of dry eye $18(42.8 \%)$ were found positive, of the 19 patients with symptoms of watering from eyes $13(68.4 \%)$ were found positive, of the 9 patients with symptoms of blepharitis
$5(55.5 \%)$ were found positive and of the 20 patients with chalazion/ hardoleum $10(50 \%)$ were found positive for $C$. trachomatis antigen with DFA (Table 1). Of the 55 males $30(54.5 \%)$ were found positive and of the 57 females $32(56.1 \%)$ were positive for $C$. trachomatis antigen with DFA. Females show slightly higher positivity compared to males ( Two tailed P- value $=0.8652$ (Chi square $=0.029$ ); 95\% CI: 30.82 - 36.82; df: 111). The value, 0.8652 , is above 0.05 so we declare the result to be statistically non-significant. This signifies that, there is no correlation between the DFA results and gender in the studied population. Patients with the symptoms of meibomitis showed highest positivity (72.7\%), whereas patients with dry eye showed least positivity (42.8\%) for C. trachomatis antigen with DFA. Chlamydia elementary bodies appeared as round, bright, apple green, fluorescent particles, regular in outline (Figure 1). However, we did not observe any significant positivity difference in different age group/sex of the patients. Of the total 62 C. trachomatis positive cases $54(87.09 \%)$ had good clinical response for topical azithromycin (1\% eye drops (twice a day) for $2-4$ weeks) and topical lubricants and warm compresses.

\section{Discussions}

The underlying mechanism of MGD with follicular conjunctival inflammation is not yet clear; and the involvement of different bacteria in the pathophysiology of MGD is still a controversial subject. As per the conventional therapy of MGD is concerned, lid massage, lid expression and treatment with tetracycline and doxycycline, were used to treat MGD and relieving the symptoms [11,12]. Some of the recent studies had reported the effectiveness of topical azithromycin therapy for meibomian gland dysfunction and improving the signs and symptoms of the disease $[11,13]$. In a pilot study it was reported that, order of the neutral and polar lipid molecules gets altered in the disease state compared to normal state and treatment with azithromycin (antibacterial agent) can improve that abnormal condition towards normal by reducing the bacterial load in the eye lid $[11,14]$. Changes in the concentration of oleic acid in the meibum results in the increase in percentage of unsaturation of fatty acid associated with phospholipids in case of meibomitis was also reported in some studies $[15,16]$. In another pilot study over blepharitis, it was reported that, azithromycin provided significant improvement in signs and symptoms of blepharitis infections of the eyes $[13,17]$.

Association of different bacteria with meibomian gland disorder have been reported earlier. It was 
suggested that, MGD may be due to the effects of some of the bacteria themselves and their toxic metabolites [6]. Azithromycin is a broad-spectrum macrolide antibiotic against Gram positive, Gram negative, and atypical bacteria species, due its effective tissue penetration property, good pharmacokinetics for daily dose and a sustained delivery mechanism [18]. Currently the drug azithromycin is used widely in treatment of ocular and genital chlamydial infections and has been used in several trachoma control programs for mass antibiotic administration $[17,18]$. Azithromycin has also potent ocular anti-inflammatory properties but the mechanism behind its anti-inflammatory property is not clear [19]. In one of the pilot study it was reported that, azithromycin suppresses the activation of nuclear factor-kappa B and synthesis of pro-inflammatory cytokinesin tracheal aspirate cells which make this antibiotic more effective than other antibiotics in treating the bacterial infections [20]. Besides this fact, azithromycin suppresses zymosaninduced production of pro inflammatory mediators by human corneal epithelial cells [21]. Currently trachoma prevalence in Indian population is $\sim 6 \%$, but in this group of meibomitis patients C. trachomatis was detected in $55 \%$ of patients signifying a possible role of $C$. Trachomatis in MGD with follicular conjunctival inflammation [22-24].

In sporadic follicular conjunctivitis patients $C$. trachomatis antigen detection rate varied between 2228\% [25]. Moreover the symptoms were relieved after azithromycin treatment in $87 \%$ of the patients thereby suggesting a possible association with $C$. trachomatis infections. Meibomian glands are the oil glands present in the margin of the eyelids. In a healthy individual secretion of meibomian gland consists of mainly neutral sterols and wax esters (nonpolar lipids), with lesser amounts of polar lipids, triglycerides, diesters, triesters, and free sterols [15]. Several changes had been observed in the meibomian lipid composition which result in MGD that includes, increased monounsaturated fatty acids (wax and sterol ester fatty acids) or other fatty acids [26,27]. Bacterial isolates found in MGD produce lipases that can alter the lipid composition which may in turn, enhance the growth of other microorganisms $[26,8]$.

All these changes in the meibomian gland secretion results in abnormal meibum secretion from gland having higher melting temperature which results in thicker meibum, stagnation, ductal plugging, and pouting of the meibomian gland orifices resulting in meibomian gland dysfunction [28]. In previous studies topical azithromycin therapy relieved signs and symptoms of MGD and restored the lipid properties of the abnormal meibomian gland secretion [11]. In this study since $C$. trachomatis was detected in more than half of the patients, it suggests a possible association.

\section{Conclusion}

We concluded from this pilot study that, $C$. trachomatis is supposed to be one of the major pathogenic bacteria for MGD with follicular conjunctival inflammation and $1 \%$ topical azithromycin (given as anti-chlamydial therapy) is a good choice for the treatment and improving the signs and symptoms of the disorder. As the role of chlamydia in MGD is not yet reported, this study will disclose an exciting fact about the pathophysiology of the disease, which will in turn improve treatment strategy to some extent to combat with the common but frustrating infection.

\section{Ethical Approval}

All procedures performed in this study involving human participants were in accordance with the ethical standards of the institute.

\section{Funding Information}

There are no funding agencies to report for this submission.

\section{Acknowledgements}

Authors thank all the clinical faculty members and resident doctors of Dr R.P. Centre for Ophthalmic Sciences for sending the clinical specimens for clinical investigations.

\section{Conflicts of Interest}

The authors declare that there are no conflicts of interest related to this work.

\section{References}

1. Kelly KN, Gary NF, Anthony JB, Ben JG, Murat D, et al. (2011) The International Workshop on Meibomian Gland Dysfunction: Executive Summary. Invest Ophthalmol Vis Sci 52(4): 1922-1929.

2. Bron AJ, Tiffany JM (2004) The contribution of meibomian disease to dry eye. Ocul Surf 2(2): 149165. 
3. Viso E, Gude F, Rodriguez-Ares MT (2011) The association of meibomian gland dysfunction and other common ocular diseases with dry eye: a population-based study in Spain. Cornea 30(1): 1-6.

4. Asbell PA, Lemp MA (2007) Dry eye disease, The clinician's guide to diagnosis and treatment. New York: Thieme 2: 1-23.

5. Nelson JD, Shimazaki J, Benitez-del-Castillo J, Jennifer P, James P, et al. (2011) The international workshop on meibomian gland dysfunction: report of the definition and classification subcommittee. Invest Ophthalmol Vis Sci 52(4): 1930-1937.

6. Geerling G, Tauber J, Baudouin C, Eiki G, Yukihiro M, et al. (2011) The international workshop on meibomian gland dysfunction: report of the subcommittee on management and treatment of meibomian gland dysfunction. Invest Ophthalmol Vis Sci 52(4): 2050-2064.

7. Dougherty JM, McCulley JP (1984) Comparative bacteriology of chronic blepharitis. $\mathrm{Br} \mathrm{J}$ Ophthalmol 68(8): 524-528.

8. McCulley JP, Dougherty JM (1986) Bacterial aspects of chronic blepharitis. Trans Ophthalmol Soc U K 105(3): 314-318.

9. Iovieno A, Lambiase A, Micera A, Stampachiacchiere $\mathrm{B}$, Sgrulletta R, et al. (2009) In vivo characterization of doxycycline effects on tear metalloproteinases in patients with chronic blepharitis. Eur J Ophthalmo 19(5): 708-716.

10. Mohile M, Deorari AK, Satpathy G, Sharma A, Singh M (2002) Microbiological study of neonatal conjunctivitis with special reference to C. trachomatis. Indian J Ophthalmol 50(4): 295-299.

11. Foulks GN, Borchman D, Yappert M, Kim SH, McKay JW (2010) Topical azithromycin therapy for meibomian gland dysfunction: clinical response and lipid alterations. Cornea 29(7): 781-788.

12. Paranjpe DR, Foulks GN (2003) Therapy of meibomian gland disease. Ophthalmol Clin North Am 16: $37-42$.

13. Haque RM, Torkildsen GL, Brubaker K, Zink RC, Kowalski RP, et al. (2010) Multicenter open-label study evaluating the efficacy of azithromycin ophthalmic solution $1 \%$ on the signs and symptoms of subjects with blepharitis. Cornea 29(8): 871-877.

14. Sullivan DA, Sullivan BD, Evans JE, Schirra F, Yamagami H, et al. (2002) Androgen deficiency, Meibomian gland dysfunction, and evaporative dry eye. Ann N Y Acad Sci 966: 211-222.

15. Shine WE, McCulley JP (2003) Polar lipids in human meibomian gland secretions. Curr Eye Re 26(2): 8994.

16. Shine WE, McCulley JP (2000) Association of meibum oleic acid with meibomian seborrhea. Cornea 19(1): 72-74.

17. John T, Shah AA (2008) Use of azithromycin ophthalmic solution in the treatment of chronic mixed anterior blepharitis. Ann Ophthalmol Skokie 40(2): 68-74.

18. Friedlaender $\mathrm{MH}$, Protzko E (2007) Clinical development of $1 \%$ azithromycin in DuraSite, a topical azalide anti-infective for ocular surface therapy. Clin Ophthalmol 1(1): 3-10.

19. Qiao J, Yan X (2013) Emerging treatment options for meibomian gland dysfunction. Clin Ophthalmol 7: 1797-1803.

20. Aghai ZH, Kode A, Saslow JG, Nakhla T, Farhath S, et al. (2007) Azithromycin suppresses activation of nuclear factor-kappa B and synthesis of proinflammatory cytokines in tracheal aspirate cells from premature infants. Pediatr Res 62(4): 483-488.

21. Li DQ, Zhou N, Zhang L, Ma P, Pflugfelder SC (2010) Suppressive effects of azithromycin on zymosaninduced production of proinflammatory mediators by human corneal epithelial cells. Invest Ophthalmol Vis Sci 51(11): 5623-5629.

22. Malathi J, Madhavan HN, Therese KL, Joseph PR (2003) A hospital based study on the prevalence of conjunctivitis due to Chlamydia trachomatis. Indian J Med Res 117: 71-75.

23. Khanduja S, Jhanji V, Sharma N, Vashist P, Murthy GV, et al. (2012) Trachoma prevalence in women living in rural northern India: rapid assessment findings. Ophthalmic Epidemiol 19(4): 216-220. 
24. Malhotra S, Vashist P, Gupta N, Kalaivani M, Satpathy G, et al. (2016) Prevalence of Trachoma in CarNicobar Island, India after Three Annual Rounds of Mass Drug Administration with Azithromycin. PLoS One 11(7): e0158625.

25. Sharma A, Satpathy G, Nayak N, Tandon R, Sharma N, et al. (2012) Ocular Chlamydia trachomatis infections in patients attending a tertiary eye care hospital in north India: a twelve year study. Indian J Med Res 136(6): 1004-1010.
26. Dougherty JM, Osgood JK, McCulley JP (1991) The role of wax and sterol ester fatty acids in chronic blepharitis. Invest Ophthalmol Vis Sci 32(6): 19321937.

27. Ong BL, Larke JR (1990) Meibomian gland dysfunction: some clinical, biochemical and physical observations. Ophthalmic Physiol Opt 10(2): 144-148.

28. Henriquez AS, Korb DR (1981) Meibomian glands and contact lens wear. Br J Ophthalmol 65(2): 108-111. 